\title{
The use of intraoperative neurosurgical ultrasound for surgical navigation in low- and middle-income countries: the initial experience in Tanzania
}

\author{
Aingaya J. Kaale, MD, ${ }^{1}$ Nicephorus Rutabasibwa, MD, ${ }^{1}$ Laurent Lemeri Mchome, MD, ${ }^{1}$ \\ Kevin O. Lillehei, MD, ${ }^{2}$ Justin M. Honce, MD, ${ }^{3}$ Joseph Kahamba, MD, ${ }^{1}$ and \\ D. Ryan Ormond, MD, PhD²
}

1Division of Neurosurgery, Muhimbili Orthopaedic and Neurosurgical Institute, Muhimbili University of Health and Allied Sciences, Dar es Salaam, Tanzania; and Departments of ${ }^{2}$ Neurosurgery and ${ }^{3}$ Radiology, University of Colorado School of Medicine, Aurora, Colorado

\begin{abstract}
OBJECTIVE Neuronavigation has become a crucial tool in the surgical management of CNS pathology in higherincome countries, but has yet to be implemented in most low- and middle-income countries (LMICs) due to cost constraints. In these resource-limited settings, neurosurgeons typically rely on their understanding of neuroanatomy and preoperative imaging to help guide them through a particular operation, making surgery more challenging for the surgeon and a higher risk for the patient. Alternatives to assist the surgeon improve the safety and efficacy of neurosurgery are important for the expansion of subspecialty neurosurgery in LMICs. A low-cost and efficacious alternative may be the use of intraoperative neurosurgical ultrasound. The authors analyze the preliminary results of the introduction of intraoperative ultrasound in an LMIC setting.
\end{abstract}

METHODS After a training program in intraoperative ultrasound including courses conducted in Dar es Salaam, Tanzania, and Aurora, Colorado, neurosurgeons at the Muhimbili Orthopaedic and Neurosurgical Institute began its independent use. The initial experience is reported from the first 24 prospective cases in which intraoperative ultrasound was used. When possible, ultrasound findings were recorded and compared with postoperative imaging findings in order to establish accuracy of intraoperative interpretation.

RESULTS Of 24 cases of intraoperative ultrasound that were reported, $29.2 \%$ were spine surgeries and $70.8 \%$ were cranial. The majority were tumor cases $(95.8 \%)$. Lesions were identified through the dura mater in all 24 cases, with $20.8 \%$ requiring extension of craniotomy or laminectomy due to inadequate exposure. Postoperative imaging (typically CT) was only performed in 11 cases, but all 11 matched the findings on post-dural closure ultrasound.

CONCLUSIONS The use of intraoperative ultrasound, which is affordable and available locally, is changing neurosurgical care in Tanzania. Ultimately, expanding the use of intraoperative B-mode ultrasound in Tanzania and other LMICs may help improve neurosurgical care in these countries in an affordable manner.

https://thejns.org/doi/abs/10.3171/2019.12.JNS192851

KEYWORDS intraoperative ultrasound; neuronavigation; low- and middle-income countries; Tanzania; surgical technique

$\mathrm{N}$ EUROSURGERY has long been dependent on imaging for diagnostic and intraoperative navigation assistance. In the early days of neurosurgery, the use of angiography and pneumoencephalography helped localize lesions better than physical examination alone. Later on, CT and MRI were developed and were quickly coupled to stereotactic frames and navigation systems to help improve intraoperative brain localization. Image-guided navigation systems have now become a routine tool in neurosurgery to assist in the intraoperative management of neurosurgical diseases. ${ }^{24}$ All of these systems, however, continue to have limitations. In the spine, localization is mainly driven by $\mathrm{CT}$ and does not demonstrate soft tissue well. In the brain, images are affected by brain shift: parenchymal de-

ABBREVIATIONS GTR = gross-total resection; LMICs = low- and middle-income countries; $\mathrm{MOI}=$ Muhimbili Orthopaedic and Neurosurgical Institute; UCSOM = University of Colorado School of Medicine.

SUBMITTED October 19, 2019. ACCEPTED December 30, 2019.

INCLUDE WHEN CITING Published online February 28, 2020; DOI: 10.3171/2019.12.JNS192851. 
formation occurring due to swelling, gravity, CSF egress, surgical manipulation, etc. ${ }^{7,19,32}$ This makes the image localization less accurate over time. Additional options have been developed to overcome these challenges. Intraoperative MRI and CT, for example, have been introduced to allow an update of information intraoperatively, but come with their own benefits and limitations, like cost and time for implementation.

Although neuronavigation has taken hold as a routine modality in higher-income countries, it has yet to be implemented in most low- and middle-income countries (LMICs) due to cost constraints. In these resource-limited settings, neurosurgeons typically rely on their understanding of neuroanatomy and preoperative imaging to help guide them through a particular surgery. Biopsies in these settings can be challenging, especially biopsies of deepseated lesions in the brain. Identification of tumor borders, especially in diffuse gliomas, can be even more challenging without navigational adjuncts to surgery. In intradural spine surgery without imaging adjuncts at surgery, the operation occasionally has to be aborted, or the spinal cord may be entered and no lesion identified. All of this puts the patient at increased risk of neurological deficit. Without access to CT- or MR-based navigation systems, alternatives to help the surgeon improve the safety and efficacy of neurosurgery are important for the expansion of subspecialty neurosurgery in LMICs. The use of intraoperative neurosurgical ultrasound is a low-cost and efficacious alternative that may prove useful in this setting.

Intraoperative ultrasound has been used for several decades, actually predating many other navigation systems. ${ }^{11,12,17,18,33}$ It allows real-time image guidance throughout a procedure without the costs or time constraints of other more expensive and sophisticated neuronavigation systems, and has been shown to potentially be as good as or better than any other neurosurgical navigation modality, depending on pathology. ${ }^{16,33,34}$ Unfortunately, a number of limitations have prevented its widespread use. Spatial resolution, orientation of the field of view (different from standard orthogonal planes), scan quality (largely operator dependent), and lack of neurosurgeon experience have all resulted in ultrasound often being supplanted by CT- and MR-based navigation systems. ${ }^{21,22}$ However, in the hands of an experienced sonographer intraoperative ultrasound can play a pivotal role in improving surgical safety and efficacy for the patient.

In LMICs, where the majority of hospitals have no access to neurosurgical navigation systems, they do have access to ultrasound systems. This is the case in Tanzania, where neurosurgeons have previously relied on preoperative imaging and the surgeon's knowledge of neuroanatomy to guide surgery. However, ultrasound machines are available in our hospital, with existing infrastructure for maintenance and support. With this in mind, and in collaboration with the University of Colorado School of Medicine (UCSOM) Department of Neurosurgery, we developed a plan to introduce intraoperative ultrasound to neurosurgery in Tanzania. Herein, we describe the development of an intraoperative ultrasound course for the training of neurosurgeons in an LMIC, and the creation of faculty and resident exchanges for the further develop- ment of skills using ultrasound. We also present the initial experience after the adoption of intraoperative ultrasound in neurosurgery at the Muhimbili Orthopaedic and Neurosurgical Institute (MOI) in Dar es Salaam, Tanzania.

\section{Methods}

This research was performed according to the Declaration of Helsinki, following international ethical standards for clinical research, and with the approval of the local ethics committee at the Muhimbili University of Health and Allied Sciences.

Under the auspices of a memorandum of understanding set up in 2016 between the MOI Division of Neurosurgery and the UCSOM Department of Neurosurgery, ${ }^{20}$ clinical observerships were set up for faculty and residents from MOI to come to UCSOM. During these 1-month subspecialty observerships, time was devoted for MOI faculty and residents to work in radiology observing neonatal cranial ultrasound studies, along with exposure to the use of intraoperative ultrasound in cranial and spine surgery at UCSOM. Following this experience, an annual course at MOI in intraoperative ultrasound was instituted beginning in June 2018. This course was taught by neurosurgery and radiology faculty from MOI and UCSOM. Thereafter, MOI faculty began the independent use of intraoperative ultrasound. Cases were recorded prospectively, with details regarding whether ultrasound use was successful at the time of surgery and what was gained by the use of ultrasound for each individual case. If cases were altered due to imaging findings, this was also recorded. Additional details of training methods follow.

\section{Collaboration With Radiology}

Radiology has been a key player in the training of MOI neurosurgeons. Collaboration at UCSOM and MOI enabled the practical experience in pediatric cranial ultrasound at both institutions and was also instrumental in development of the local hands-on ultrasound course at MOI. Local radiologists at MOI have also continued collaborating with the neurosurgeons, accompanying them into the operating room during intraoperative ultrasound use to assist with anatomy and pathology interpretation.

\section{Faculty and Resident Exchange}

Since 2016, a faculty and resident exchange has occurred frequently between MOI and UCSOM. As MOI staff came to Colorado, subspecialty observerships were established with topics selected by the visiting surgeons. In addition, a course in pediatric and intraoperative ultrasound was arranged for all visiting individuals. Pediatric cranial ultrasound consisted of a PDF-based course syllabus of cranial ultrasound anatomy and basic pathology, and 1-2 days per week over the 1-month observership were devoted to rotating with radiology technicians performing pediatric cranial ultrasound, as well as interacting with neuroradiologists interpreting imaging results. Intraoperative ultrasound was also performed routinely in the operating rooms by neurosurgeons multiple days per week, allowing the observation of clinical setup and use of ultrasound for cranial and spine surgery. Pathologies 
witnessed included hemorrhages, tumors, and infectious processes such as brain abscesses.

\section{Course Description}

Beginning in 2018, a local, hands-on intraoperative neurosurgical ultrasound course has been held annually at MOI with faculty from neurosurgery and radiology from MOI and UCSOM. Topics have included basic ultrasound history, physics, and semiology; normal pediatric cranial ultrasound anatomy; basic adult brain semiology and surgical anatomy; basic adult spine semiology and surgical anatomy; intraoperative ultrasound for cranial pathology (tumors, infection, hematoma, hydrocephalus); intraoperative ultrasound for spine/peripheral nerve pathology; and a practical discussion of the challenges of implementation and interpretation at MOI, with didactic sessions covering 4 hours of lecture. Practical experience included surgical cases using intraoperative ultrasound for brain and spine pathology as well as experience in pediatric cranial ultrasound. Specifically, pediatric patient volunteers with normal and abnormal brain anatomy were available for an entire afternoon of practical experience, allowing course participants the opportunity to use ultrasound to identify normal and abnormal intracranial anatomy. Two additional days were spent in intraoperative demonstrations of ultrasound use for cranial and spine surgery at MOI. Radiology and neurosurgery personnel participated in both the pediatric cranial ultrasound and intraoperative cases.

\section{Equipment Procurement, Maintenance, and Setup}

The departments of radiology and anesthesia both have acquired ultrasound machines at MOI. MOI sees approximately 1500 cases of pediatric hydrocephalus annually, giving radiology a significant experience with pediatric cranial ultrasound. Maintenance and repair of ultrasound machines is performed by staff housed within the radiology department, although bioengineering support remains limited. Setup in the operating room for intraoperative ultrasound was accomplished with the use of the machine acquired by the anesthesia department. A fabric sleeve was manufactured and sterilized locally for use along the cable of the ultrasound probe. This was attached to a sterile glove that was placed over the ultrasound probe and attached to the sleeve with the use of sterile rubber bands. The ultrasound was then placed opposite the craniotomy or opposite the lead surgeon in a spine case. An experienced sonographer was present in the operating room from the department of radiology, and performed any manual control changes necessary, while the surgeon controlled the ultrasound probe (as shown during a cranial case during the practical course in Fig. 1).

\section{Inauguration of Intraoperative Ultrasound at $\mathrm{MOI}$}

After multiple neurosurgical faculty members from MOI completed ultrasound instruction at UCSOM and the inaugural ultrasound course had been completed in 2018 at MOI, intraoperative ultrasound was used by MOI neurosurgeons in collaboration with MOI radiology faculty. Our initial experience is reported.

\section{Results}

All reported cases of intraoperative ultrasound at MOI performed between July 1, 2018, and August 2, 2019, are listed in Table $1(n=24)$. Of these cases, $29.2 \%$ were spine surgeries and $70.8 \%$ were cranial. The majority of cases in which ultrasound was used were tumor cases (95.8\%). The remaining case was a patient with a cervicothoracic syrinx undergoing syringosubarachnoid shunt insertion. The goal of every case was for ultrasound to assist in the following: 1) localizing the lesion prior to dural opening; 2) guiding the corticectomy or myelotomy (as indicated); 3 ) evaluating the extent of resection; and 4) assessing the resection cavity after dural closure. The benefit of intraoperative ultrasound was divided into the 4 stages noted above for review. In all cases ultrasound was successfully performed through all stages of the procedure without technical difficulty, although in 2 cases staff failed to record post-dural closure ultrasound outcomes information. The large size of the available linear and curved phase array probes available was challenging in some areas where there is a relatively small sonographic window for visualization; e.g., the posterior fossa and the spine.

Prior to opening the dura mater (all cases involved intradural work), ultrasound was used to ensure adequate lesion exposure. Lesions were identified through the dura in all 24 cases, with $20.8 \%$ of cases requiring extension of the craniotomy or laminectomy due to inadequate exposure. Eight cases were intradural extramedullary lesions (see Table 1). Ultrasound was instructive in preparing myelotomy/corticectomy in all 16 intramedullary masses. Postoperative imaging (typically CT) was performed in only 11 cases, but all 11 matched the findings on post-dural closure ultrasound; i.e., 6 confirmed gross-total resection (GTR) and 5 confirmed residual tumor with no postoperative large-volume hematoma. The remaining 13 patients could not afford postoperative CT or MRI, although 11 received a post-dural closure ultrasound (either not performed or findings not recorded in 2 cases). Of these 11 patients, 10 demonstrated GTR with no significant postoperative hemorrhage and 1 showed an expected residual with no hemorrhage. Figure 2 shows ultrasound visualization of a spinal cord lesion and associated syrinx. The tumor was resected with syrinx decompression.

\section{Discussion}

The advent of neuronavigation has made a significant difference in anatomical accuracy and safety in neurosurgery, improving outcomes for many patients. Unfortunately, CT- and MRI-based neuronavigation is not readily available in LMICs due to the cost constraints associated with these systems. Brain shift after craniotomy can also lead to a number of anatomical inaccuracies with neuronavigation, at times causing a shift of more than 5-10 mm. ${ }^{15,25}$ Intraoperative systems to correct for brain shift are rare and even more cost prohibitive. Intraoperative ultrasound overcomes many of these challenges, in that it is a portable system that offers live imaging. It does not require its own operating theater suite, ${ }^{1}$ and is significantly cheaper and therefore much more affordable for LMICs' hospitals to acquire and maintain. Herein we have de- 


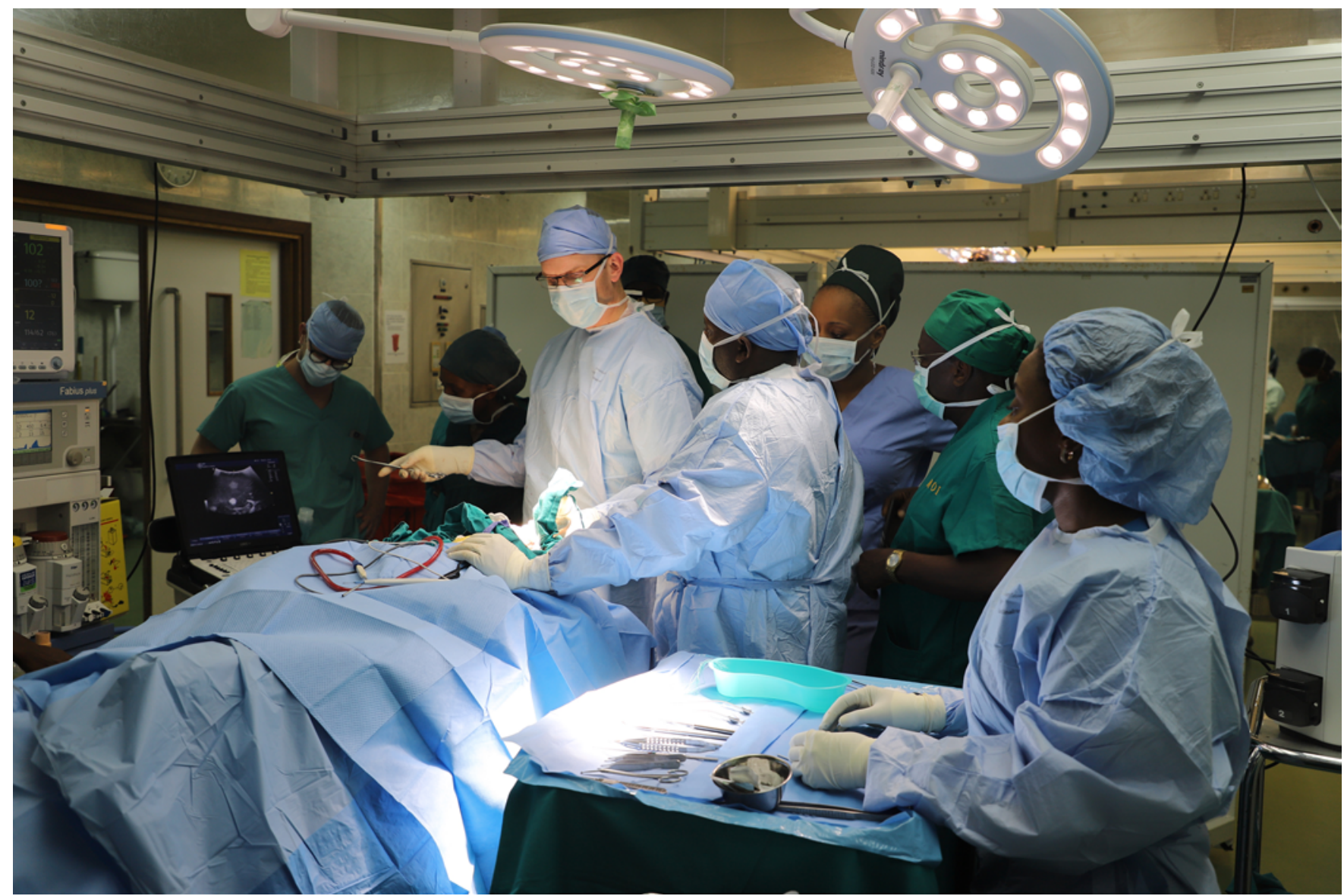

FIG. 1. Intraoperative setup of the ultrasound machine at $\mathrm{MOI}$ and demonstration of its use for a cranial case during a recent ultrasound course. Figure is available in color online only.

scribed the successful early experience of the integration of intraoperative ultrasound in brain and spine tumor surgery in Tanzania.

\section{Ultrasound in Neurosurgery}

The use of ultrasound to improve the navigation and safe access to and resection of brain and spinal lesions, to access deep-seated structures for biopsy, or to find difficult-to-access ventricles has been implemented since the $1980 \mathrm{~s} .{ }^{8}$ It has been frequently used for the resection of brain tumors. Previous studies have shown that most brain tumors are visible with intraoperative ultrasound. ${ }^{16,33-36} \mathrm{In}$ fact, extent of resection and even survival have been noted to improve with the use of intraoperative ultrasound. ${ }^{27,31,37}$ The positive predictive value of ultrasound in detecting residual tumor has been shown to be as high as $0.84 .^{2}$ Rygh et al. demonstrated a sensitivity and specificity as high as $95 \%$, while acknowledging that during resection, ultrasound specificity decreased to $42 \%$, although sensitivity remained high $(87 \%) .{ }^{26}$

Additional technologies have improved the accuracy of intraoperative ultrasound. Basic B-mode ultrasound typically includes 3 small probes that are easily introduced into the surgical field. ${ }^{17,18} \mathrm{~A}$ linear array at higher frequency $(8-15 \mathrm{MHz})$ has a limited field of view but depicts su- perficial lesions $(1-4 \mathrm{~cm})$ with high resolution. A curved linear array (2-12 MHz) displays intermediate lesions $(2-6 \mathrm{~cm})$ well. A sector (phased) array is best for deep lesions $(4-8 \mathrm{~cm})$ and works at lower frequency $(2-6 \mathrm{MHz}){ }^{17}$ Additional developing technologies such as contrast-enhanced ultrasound, $, 9,13,22$ strain elastography, 3,4,28,29 shear wave elastography, ${ }^{5,10,28}$ navigated 3D ultrasound with or without MRI or CT fusion, ${ }^{6,22}$ and Doppler for blood flow ${ }^{6}$ are additional adjuncts to standard B-mode intraoperative ultrasound that enhance the value of ultrasound as an intraoperative diagnostic tool. In addition, focused ultrasound may play a role in the treatment of cerebrovascular diseases and brain tumors. ${ }^{23}$ Overall, ultrasound has a strong and growing presence and value in the management of neurosurgical diseases.

\section{Benefits of Intraoperative Ultrasound at MOI}

A number of early benefits have already been realized using intraoperative ultrasound at MOI. Ultrasound scanning prior to opening the dura provides an increased level of confidence that the lesion is visible and can be readily accessed. Postoperative imaging was frequently able to confirm the degree of resection as indicated by ultrasound, demonstrating the accuracy of the technique at MOI. The assistance of radiology in the operating room (already ex- 


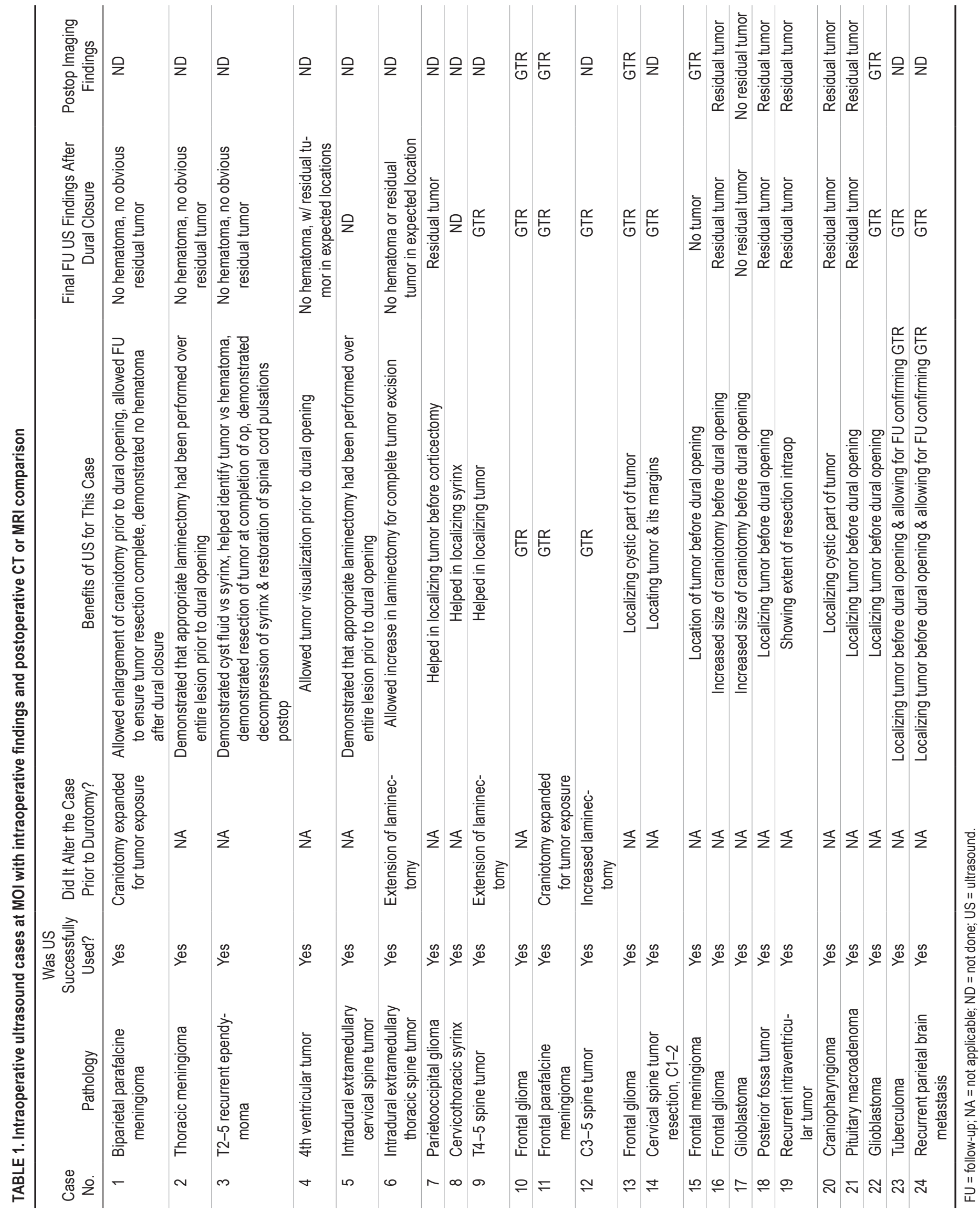



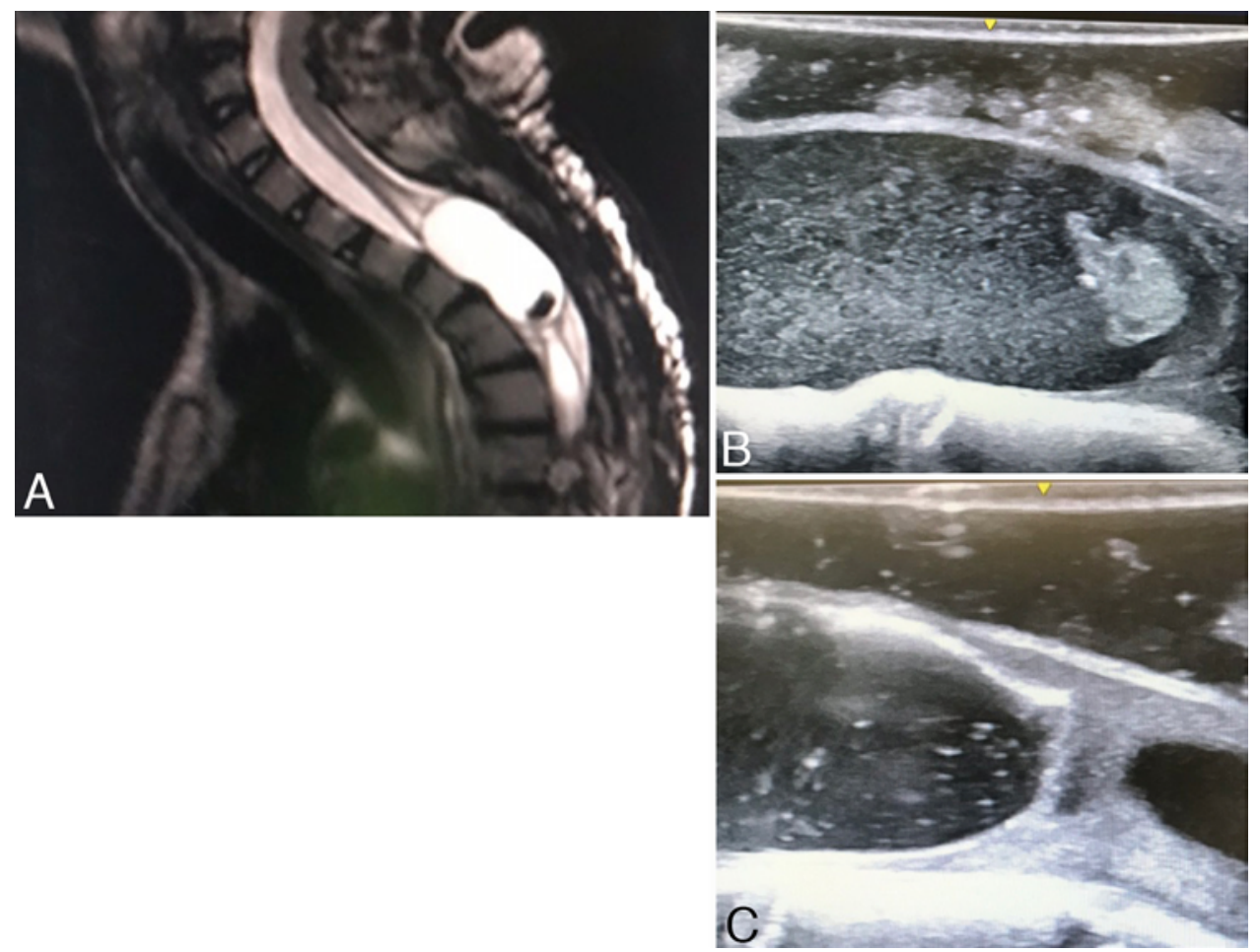

FIG. 2. A: Preoperative T2-weighted sagittal cervical spine image demonstrating an upper thoracic recurrent spinal cord ependymoma with associated cyst and syrinx. The patient presented with progressive neurological decline. B: Intraoperative ultrasound demonstrating a cyst with an associated nodule. C: After dural closure, ultrasound demonstrates the decompressed cyst with nodule resected completely. Figure is available in color online only.

perienced in the use of pediatric cranial ultrasound and interpretation at MOI) greatly improved ultrasound use and interpretation, helping to reduce the steep learning curve for this technology. Unique to this patient population, the use of ultrasound provided a postresection control image. This proved useful, because some patients could not afford traditional CT or MRI after surgery due to cost constraints. Previously, these patients would have had no postoperative imaging. Despite the learning curve of intraoperative ultrasound, neurosurgeons are already finding benefit from the adoption of this new technology.

\section{Limitations of Ultrasound}

Although intraoperative ultrasound has proven efficacy in several studies, and it is quite cost-effective and efficient, ${ }^{1}$ its use remains limited in much of the neurosurgical world. This is probably due in part to past experiences when B-mode image resolution was poor, and to the steep learning curve associated with proper interpretation of imaging findings, especially as surgery progresses. Ultrasound has a restricted field of view, and typically does not display traditional orthogonal views like CT and MRI, making it difficult to interpret without experience. ${ }^{1}$ Interpretation of ultrasound images is quite user dependent, and it can be difficult to distinguish between edema and tumor, especially in the setting of hypoechogenic lesions or changes that occur during surgery (hemorrhage, use of hemostatic material, increasing edema, expanding cavity changing ultrasound characteristics at the margins, etc.). 1,14,17,22,24 These changes can be minimized, and the most important way to overcome these challenges is experience. ${ }^{30}$ An understanding of normal and pathological ultrasound anatomy in the brain and spine, and how this anatomy changes over the course of surgery, is critical to an understanding of intraoperative ultrasound. Additionally, future improvements in ultrasound probe technology and research into image analysis will help to overcome the limitations, allowing maximum benefit of intraoperative ultrasound for the neurosurgical patient.

\section{Specific Limitations of Ultrasound at MOI, and Future Directions}

Currently the use of intraoperative ultrasound at MOI is limited by available technology on site. Neurosurgery relies on a B-mode ultrasound machine donated to the anesthesia department. Although producing good-quality images and being relatively simple to use, the probes are larger than ideal for neurosurgical use, and there is no burr hole probe for minimally invasive applications like biopsy, ventricular access, or abscess/cyst aspiration. Advanced ultrasound technology is also not yet available. Lack of standard ultrasound drapes sometimes has resulted in a need for redraping during operations, prolonging the procedure. No neurosurgeon at MOI had significant prior experience with intraoperative ultrasound, so the learning curve has been steep.

In order to accommodate and accelerate this learning to make intraoperative ultrasound effective at MOI, a 
multimodality approach has been developed to facilitate training and experience. ${ }^{1}$ Faculty and residents participated in observerships at UCSOM to gain experience with intraoperative and pediatric cranial ultrasound at a facility where it is already in routine use. While informal and ad hoc, these experiences gave early and valuable experience for MOI surgeons regarding anatomy and pathology recognizable by ultrasound and the benefits and limitations of its use in common practice. Local courses on site at MOI facilitated by UCSOM faculty and MOI radiologists helped further facilitate education regarding anatomical and pathological imaging with ultrasound machines available locally. Collaboration with radiology has been instrumental in these efforts, because local radiologists can help pass on their understanding of ultrasound-based anatomy and pathology to neurosurgeons and help with intraoperative interpretation of images. Existing experience at the hospital for the care and use of ultrasound hardware has also been important, allowing for the more rapid adoption of this technology (i.e., only training of neurosurgeons was necessary for full implementation). Finally, frequent use of ultrasound in routine cases to better understand normal anatomy and pathology and how it changes over the course of surgery will help increase the utility of intraoperative ultrasound in Tanzania.

\section{Conclusions}

Without the use of intraoperative navigation, neurosurgeons at MOI have previously relied solely on preoperative imaging and anatomical understanding for operative intervention. The use of intraoperative ultrasound, which is affordable and available locally, is changing neurosurgical care in Tanzania. Ultimately, expanding the use of intraoperative B-mode ultrasound in Tanzania and other LMICs may help improve neurosurgical care in these countries in an affordable manner.

\section{References}

1. Bal J, Camp SJ, Nandi D: The use of ultrasound in intracranial tumor surgery. Acta Neurochir (Wien) 158:1179-1185, 2016

2. Chacko AG, Kumar NK, Chacko G, Athyal R, Rajshekhar V: Intraoperative ultrasound in determining the extent of resection of parenchymal brain tumours - a comparative study with computed tomography and histopathology. Acta Neurochir (Wien) 145:743-748, 2003

3. Chakraborty A, Bamber J, Dorward N: Preliminary investigation into the use of ultrasound elastography during brain tumour resection. Ultrasound 20:33-40, 2012

4. Chan HW, Pressler R, Uff C, Gunny R, St Piers K, Cross H, et al: A novel technique of detecting MRI-negative lesion in focal symptomatic epilepsy: intraoperative ShearWave elastography. Epilepsia 55:e30-e33, 2014

5. Chauvet D, Imbault M, Capelle L, Demene C, Mossad M, Karachi C, et al: In vivo measurement of brain tumor elasticity using intraoperative shear wave elastography. Ultraschall Med 37:584-590, 2016

6. Del Bene M, Perin A, Casali C, Legnani F, Saladino A, Mattei L, et al: Advanced ultrasound imaging in glioma surgery: beyond gray-scale B-mode. Front Oncol 8:576, 2018

7. Dorward NL, Alberti O, Velani B, Gerritsen FA, Harkness WF, Kitchen ND, et al: Postimaging brain distortion: magnitude, correlates, and impact on neuronavigation. J Neurosurg 88:656-662, 1998
8. el Mouaaouy A, Gawlowski J, Schrot G, Arlt R: [Intraoperative ultrasound diagnosis in neurosurgery.] Ultraschall Med 7:235-238, 1986 (German)

9. Engelhardt M, Hansen C, Eyding J, Wilkening W, Brenke C, Krogias C, et al: Feasibility of contrast-enhanced sonography during resection of cerebral tumours: initial results of a prospective study. Ultrasound Med Biol 33:571-575, 2007

10. Ferraioli G, Parekh P, Levitov AB, Filice C: Shear wave elastography for evaluation of liver fibrosis. J Ultrasound Med 33:197-203, 2014

11. Gerganov VM, Samii A, Akbarian A, Stieglitz L, Samii M, Fahlbusch R: Reliability of intraoperative high-resolution 2D ultrasound as an alternative to high-field strength MR imaging for tumor resection control: a prospective comparative study. J Neurosurg 111:512-519, 2009

12. Hammoud MA, Ligon BL, elSouki R, Shi WM, Schomer DF, Sawaya R: Use of intraoperative ultrasound for localizing tumors and determining the extent of resection: a comparative study with magnetic resonance imaging. J Neurosurg 84:737-741, 1996

13. He W, Jiang XQ, Wang S, Zhang MZ, Zhao JZ, Liu HZ, et al: Intraoperative contrast-enhanced ultrasound for brain tumors. Clin Imaging 32:419-424, 2008

14. Hervey-Jumper SL, Berger MS: Role of surgical resection in low- and high-grade gliomas. Curr Treat Options Neurol 16:284, 2014

15. Hill DL, Maurer CR Jr, Maciunas RJ, Barwise JA, Fitzpatrick JM, Wang MY: Measurement of intraoperative brain surface deformation under a craniotomy. Neurosurgery 43:514-528, 1998

16. LeRoux PD, Winter TC, Berger MS, Mack LA, Wang K, Elliott JP: A comparison between preoperative magnetic resonance and intraoperative ultrasound tumor volumes and margins. J Clin Ultrasound 22:29-36, 1994

17. Moiyadi AV: Intraoperative ultrasound technology in neurooncology practice-current role and future applications. World Neurosurg 93:81-93, 2016

18. Moiyadi AV: Objective assessment of intraoperative ultrasound in brain tumors. Acta Neurochir (Wien) 156:703704, 2014

19. Nimsky C, Ganslandt O, Cerny S, Hastreiter P, Greiner G, Fahlbusch R: Quantification of, visualization of, and compensation for brain shift using intraoperative magnetic resonance imaging. Neurosurgery 47:1070-1080, 2000

20. Ormond DRKJ, Kahamba J, Lillehei KO, Rutabasibwa N: Overcoming barriers to neurosurgical training in Tanzania: international exchange, curriculum development, and novel methods of resource utilization and subspecialty development. Neurosurg Focus 45(4):E6, 2018

21. Pasto ME, Rifkin MD: Intraoperative ultrasound examination of the brain: possible pitfalls in diagnosis and biopsy guidance. J Ultrasound Med 3:245-249, 1984

22. Prada F, Del Bene M, Mattei L, Casali C, Filippini A, Legnani F, et al: Fusion imaging for intra-operative ultrasoundbased navigation in neurosurgery. J Ultrasound 17:243-251, 2014

23. Prada F, Kalani MYS, Yagmurlu K, Norat P, Del Bene M, DiMeco F, et al: Applications of focused ultrasound in cerebrovascular diseases and brain tumors. Neurotherapeutics 16:67-87, 2019

24. Prada F, Perin A, Martegani A, Aiani L, Solbiati L, Lamperti M, et al: Intraoperative contrast-enhanced ultrasound for brain tumor surgery. Neurosurgery 74:542-552, 2014

25. Roberts DW, Hartov A, Kennedy FE, Miga MI, Paulsen KD: Intraoperative brain shift and deformation: a quantitative analysis of cortical displacement in 28 cases. Neurosurgery 43:749-760, 1998

26. Rygh OM, Selbekk T, Torp SH, Lydersen S, Hernes TA, Unsgaard G: Comparison of navigated 3D ultrasound findings 
with histopathology in subsequent phases of glioblastoma resection. Acta Neurochir (Wien) 150:1033-1042, 2008

27. Sæther CA, Torsteinsen M, Torp SH, Sundstrøm S, Unsgård $\mathrm{G}$, Solheim O: Did survival improve after the implementation of intraoperative neuronavigation and 3D ultrasound in glioblastoma surgery? A retrospective analysis of 192 primary operations. J Neurol Surg A Cent Eur Neurosurg 73:73-78, 2012

28. Sebag F, Vaillant-Lombard J, Berbis J, Griset V, Henry JF, Petit P, et al: Shear wave elastography: a new ultrasound imaging mode for the differential diagnosis of benign and malignant thyroid nodules. J Clin Endocrinol Metab 95:5281-5288, 2010

29. Selbekk T, Brekken R, Indergaard M, Solheim O, Unsgård G: Comparison of contrast in brightness mode and strain ultrasonography of glial brain tumours. BMC Med Imaging 12:11, 2012

30. Selbekk T, Jakola AS, Solheim O, Johansen TF, Lindseth F, Reinertsen I, et al: Ultrasound imaging in neurosurgery: approaches to minimize surgically induced image artefacts for improved resection control. Acta Neurochir (Wien) 155:973-980, 2013

31. Serra C, Stauffer A, Actor B, Burkhardt JK, Ulrich NH, Bernays RL, et al: Intraoperative high frequency ultrasound in intracerebral high-grade tumors. Ultraschall Med 33:E306E312, 2012

32. Stieglitz LH, Fichtner J, Andres R, Schucht P, Krähenbühl AK, Raabe A, et al: The silent loss of neuronavigation accuracy: a systematic retrospective analysis of factors influencing the mismatch of frameless stereotactic systems in cranial neurosurgery. Neurosurgery 72:796-807, 2013

33. Unsgaard G, Gronningsaeter A, Ommedal S, Nagelhus Hernes TA: Brain operations guided by real-time two-dimensional ultrasound: new possibilities as a result of improved image quality. Neurosurgery 51:402-412, 2002

34. Unsgaard G, Ommedal S, Muller T, Gronningsaeter A, Nagelhus Hernes TA: Neuronavigation by intraoperative three-dimensional ultrasound: initial experience during brain tumor resection. Neurosurgery 50:804-812, 2002
35. Unsgaard G, Rygh OM, Selbekk T, Müller TB, Kolstad F, Lindseth F, et al: Intra-operative 3D ultrasound in neurosurgery. Acta Neurochir (Wien) 148:235-253, 2006

36. Unsgaard G, Selbekk T, Brostrup Müller T, Ommedal S, Torp SH, Myhr G, et al: Ability of navigated 3D ultrasound to delineate gliomas and metastases-comparison of image interpretations with histopathology. Acta Neurochir (Wien) 147:1259-1269, 2005

37. Woydt M, Krone A, Becker G, Schmidt K, Roggendorf W, Roosen K: Correlation of intra-operative ultrasound with histopathologic findings after tumour resection in supratentorial gliomas. A method to improve gross total tumour resection. Acta Neurochir (Wien) 138:1391-1398, 1996

\section{Disclosures}

The authors report no conflict of interest concerning the materials or methods used in this study or the findings specified in this paper.

\section{Author Contributions}

Conception and design: Ormond. Acquisition of data: Kaale, Rutabasibwa, Mchome. Analysis and interpretation of data: all authors. Drafting the article: Ormond, Kaale. Critically revising the article: all authors. Reviewed submitted version of manuscript: all authors. Approved the final version of the manuscript on behalf of all authors: Ormond. Statistical analysis: Ormond. Study supervision: Ormond.

\section{Correspondence}

D. Ryan Ormond: University of Colorado School of Medicine, Aurora, CO.david.ormond@cuanschutz.edu. 